\title{
Editorial
}

\section{Präoperatives Staging des Rectumcarcinoms mit der Computertomographie}

\author{
F. Stelzner \\ Chirurgische Universitätsklinik (Direktor: Prof. Dr. Dr. h.c. Stelzner), \\ Sigmund-Freud-Straße 25, D-5300 Bonn-Venusberg
}

Die Arbeit ist sehr sorgfältig erstellt und sie bestätigt die Ergebnisse anderer Autoren, die im Schrifttumsverzeichnis zitiert sind. Die Wertung einer neuen diagnostischen Methode muß unser bereits vorhandenes Wissen - hier beim Mastdarmkrebs - berücksichtigen. Das CT kann leider nicht entscheiden, ob eine Struktur entzündlich, narbig oder tumorös abgebildet wird. Erinnert sei an das CT nach einer Rectumamputation, wo ein Narbenblock vor dem Kreuzbein oft als rückfälliger Tumor angesprochen wird. Die gleiche Unsicherheit gilt für vergrößerte Lymphknoten im Beckenbereich. Entzündliche Reaktionen - sie sind nicht häufig -, die neben einem Mastdarmkrebs oder in den Lymphknoten der Metastasenstraße auftreten, sind prognostisch günstig. Echte Tumoreinbrüche über die Mastdarmwand hinaus sind natürlich ungünstig. Einen Hinweis für die Gefährlichkeit eines Tumors liegt auch in seiner Histologie beschlossen. Sie definiert die Krebsrasse. Hochdifferenzierte Tumoren - Zeitlupentumoren sind wesentlich besser heilbar als undifferenzierte - Zeitrafferkrebse.

Eine Auskunft darüber hätten wir in diesem Krankengut als sehr nützlich empfunden. Ein Stadium nach Dukes ist nur postoperativ - am herausgenommenen Präparat - definierbar. Deshalb ist es nicht verwunderlich, da $B$ in Tabelle 4 der vorliegenden Arbeit der präoperative Versuch, ein Dukes-Stadium vorauszusagen, am Präparat oft korrigiert werden mußte. Dukes war Pathologe! Zirkulär wachsende Mastdarmkrebse sind immer fortgeschritten. In dem vorgelegten Krankengut sind es ungefähr $25 \%$ aller Fälle. Diese Krebse gehören immer mittleren, hohen und höchsten Malignitätsstufen an. Polypöse Mastdarmkrebse, also hochdifferenzierte Geschwülste, lassen eine Zone unveränderter Mucosa neben sich (Zottentumoren können zwar zirkulär wachsen, sie sind aber keine echten Krebse. Werden sie sehr groß, können sie punktuell ein echtes Carcinom in sich tragen). 
Die Autoren bestätigen eine rätselhafte Eigentümlichkeit des Mastdarmcarcinoms, auf die wir schon oft hingewiesen haben. $81 \%$ ihrer Tumoren wachsen an der Vorder- und an der Rückwand des Mastdarms. Wir fanden $90 \%$ an diesen Stellen und nur ein kleiner Teil der Geschwülste entsteht primär seitlich. Dieses unerklärbare Verhalten (wo bleibt da die Bedeutung der Adenome, die überall rundum wachsen und die der endogenen Krebsnoxen?) spielt für die heute entwickelte Radikaloperation eine große Rolle; die meisten Mastdarmkrebse entstehen somit den Grenzlamellen gegenüber. Diese hauchdünnen Hüllfascien sind nur der Vorderwand und der Hinterwand des Rectums gegenüber entwickelt. Grenzlamellen sind gefäßlos und garantieren deshalb eine „tumordichte“ Verpackung der Geschwulst. Grenzlamellen werden bei jeder Radikaloperation herausgenommen. Ein seitlicher Mastdarmkrebs Stadium Dukes C, diese Kombination ist sehr selten, ist immer rezidivgeneigt, weil es eben dort keine Grenzlamellen gibt. In der Praxis hat sich gezeigt, daß neben der Excision der Grenzlamellen mit dem retrorectalen Fettlymphknotenareal die Entfernung der Metastasenstraße bis an die Aorta die modernen guten Dauerheilungsziffern mit bedingt. Die seitlichen Metastasenwege an die Beckenwand sind für den Chirurgen unerreichbar. Beim operablen Fall werden sie eigentümlicherweise nicht begangen. Es gibt nur eine einzige Metastasenstraße und dieser Hauptweg endet mit dem Ursprung der Arteria mesenterica inferior für den Operateur an der Aorta. Es gịbt aber Fälle, wo zwischen verkrebsten Lymphknoten im Beckenbereich alle Lymphknoten entlang der Metastasenstraße krebsfrei sind bis auf einen einzigen Knoten an der Aorta. Kaum einer dieser Fälle bleibt dauernd geheilt. Wir wissen demnach schon ohne CT einiges über die Individualität des Mastdarmkrebses. Wir werden jeden Krebs so radikal wie nötig operieren. Niemand wird eine Radikaloperation ablehnen, weil das CT ihre Hoffnungslosigkeit vermuten läßt; könnte es doch eine paratumoröse Entzündung sein und selbst im Stadium C und bei undifferenzierten Geschwulsten gibt es Dauerheilungen, wenn auch nur wenige.

Sicher wird es sehr lange dauern, bis der Beweis erbracht wird, ob die durch das CT gewonnene Erkenntnis sich im Therapiedauererfolg niederschlägt. Wir dürfen nicht vergessen, daß sich eine CT-Untersuchung auch finanziell niederschlägt. Trotzdem sollte diese neue Methode in der Forschung eingesetzt werden in der Hoffnung, etwas zu erfahren, was bisher noch unbekannt ist. Wir könnten uns zum Beispiel denken, daß ein CT-Befund uns hilft, den Kranken vor dem Eingriff darüber aufzuklären, wann Potenzstörungen zu erwarten sind. Aber sicher dürfte auch diese Auskunft nicht sein. 